\title{
PA-148 STRENGTHENING PRISON HEALTH SYSTEMS: FEASIBILITY AND CHALLENGES OF INTRODUCING PRISON HEALTH COMMITTEES (PRHCS) IN ZAMBIAN CORRECTIONAL FACILITIES
}

Clement Moonga, ${ }_{1}$ Chisele Chileshe, ${ }^{2}$ George Magwende, ${ }^{2}$ German Henostroza, ${ }^{3}$ Stephanie Topp1. ' CIDRZ, Zambia; '2Zambia Correctional Services, Zambia;

${ }^{3}$ University of Alabama at Birmingham, United States of America

\subsection{6/bmjgh-2016-000260.174}

Background In Zambia, prison health and health services are in a state of 'chronic emergency'. Since 2013, the Zambian Corrections Service (ZCS) partnered with Centre for Infectious Disease Research in Zambia (CIDRZ) to understand and strengthen prisoner health and access to healthcare. A key component of this work was the establishment of 11 facility-level Prison Healthcare Committees (PrHCs) comprising officer and inmate members, with a specific remit to deliver health education and provide monitoring for facility level service access. Findings presented are from operations research evaluating the feasibility of these PrHCs.

Methods Mixed qualitative methods included, in-depth interviews (11 Ministry and ZCS officials; 6 facility managers) and focus group discussions (FGDs) with members of 6 PrHCs, and 6 groups of non-PrHC-inmates in the same facilities. Memos were generated from participant observation in workshops and meetings preceding and after implementation. All activities were subject to verbal informed consent and interviews and FGDs were audio-recorded with permission.

Results Key informants were strongly supportive of PrHCs, noting potential for improved health information dissemination, strengthened preventive service-coverage, routine service monitoring and facility-level accountability. PrHC members confirmed ZCS-led training had taken place and that they had been given authority to deliver information-based health interventions and facilitate quicker referrals to primary care. The early phase of implementation (3-6 months at data collection) produced mixed accounts regarding PrHCs' capacity to fulfil other preventive services or conduct data collection. Departure of PrHC members due to transfer and/or release was the most frequently listed challenge. Conclusions These data suggest the feasibility of establishing a committee comprising both officers and inmates to address a fundamental gap in facility-level mechanisms for health information delivery and service accountability. Findings nonetheless suggest PrHCs will require iterative adjustments and ongoing problem-solving by local officials. Context-sensitive application of these principles to other settings may yield positive outcomes. 